\title{
Activity of endovesical gemcitabine in BCG-refractory bladder cancer patients: a translational study
}

\author{
R Gunelli', E Bercovich', O Nanni ${ }^{2}$, M Ballardini ${ }^{2}$, GL Frassineti ${ }^{2}, N$ Giovannini $^{2}$, M Fiori', E Pasquini ${ }^{3}$, P Ulivi ${ }^{2}$, \\ GL Pappagallo ${ }^{4}$, R Silvestrini' and W Zoli*,
}

'Department of Urology, Morgagni-Pierantoni Hospital, Forli, Italy; ${ }^{2}$ Istituto Scientifico Romagnolo per lo Studio e la Cura dei Tumori, Meldola (FC), Italy;

${ }^{3}$ Department of Oncology, Cervesi Hospital, Cattolica (RN), Italy; ${ }^{4}$ Department of Oncology, PF Calvi Hospital, Noale, Italy

Intravesical gemcitabine (Gem) has shown promising activity against transitional cell carcinomas (TCC) of the bladder, with moderate urinary toxicity and low systemic absorption. The present phase II study evaluated the activity of biweekly intravesical treatment with Gem using a scheme directly derived from in vitro preclinical studies. Patients with Bacille Calmette-Guérin (BCG) -refractory Ta G3, TI GI-3 TCC underwent transurethral bladder resection and then intravesical instillation with $2000 \mathrm{mg}$ Gem diluted in $50 \mathrm{ml}$ saline solution on days I and 3 for 6 consecutive weeks. Thirty-eight (95\%) of the 40 patients showed persistent negative post-treatment cystoscopy and cytology 6 months after Gem treatment, while the remaining 2 patients relapsed at 5 and 6 months. At a median follow-up of 28 months, recurrences had occurred in 14 patients. Among these, four had downstaged $(T)$ disease, three had a lower grade $(G)$ lesion and three had a reduction in both $T$ and $G$. Urinary and systemic toxicity was very low, with no alterations in biochemical profiles. In conclusion, biweekly instillation of Gem proved active in BCG-refractory Ta G3, TI GI-3 TCC. Our results highlight the importance of preclinical studies using in vitro systems that adequately reproduce the conditions of intravesical clinical treatment to define the best therapeutic schedule.

British Journal of Cancer (2007) 97, I499-1504. doi: I0. 1038/sj.bjc.6604074 www.bjcancer.com

Published online 6 November 2007

(c) 2007 Cancer Research UK

Keywords: endovesical instillation; bladder cancer; gemcitabine; BCG-refractory

Superficial transitional cell carcinomas (TCC) represent about twothirds of all bladder cancers at first presentation and are a heterogeneous population of tumours that do not invade the muscularis propria. The European Organization for Research and Treatment of Cancer and the European Association of Urology Guidelines have identified three risk categories of TCC (low, intermediate and high) (Oosterlinck et al, 2006).

Complete endoscopic eradication followed or not by a single intravesical instillation with various drugs is the standard therapy for patients with low-risk tumours, while drug instillation and/or Bacille Calmette-Guérin (BCG) treatment is reserved for patients with intermediate-risk tumours in an attempt to reduce or delay recurrence and progression. For patients with high-risk tumours, BCG is considered the most effective conservative treatment, and radical cystectomy is the current option for cases refractory to this immunological therapy (Sylvester et al, 2002; Oosterlinck et al, 2006).

No one drug has proven superior in terms of efficacy. Mitomycin, epidoxorubicin and doxorubicin have all shown to have beneficial effects, but the optimal instillation scheme remains to be defined (Malmstrom et al, 1999; Malmstrom, 2000). Similarly, the best treatment for patients who fail to respond to

*Correspondence: Dr W Zoli, Istituto Scientifico Romagnolo per lo Studiio e la Cura dei Tumori, Via P. Maroncelli 40, 47014 Meldola (FC), Italy; E-mail: w.zoli@ausl.fo.it

Revised 6 September 2007; accepted 9 October 2007; published online 6 November 2007 adjuvant intravesical treatment needs further investigation, but cytotoxic drugs could represent an interesting alternative to standard BCG therapy. In this setting, Gemcitabine (Gem), a new generation deoxycytidine analogue, given systemically as a single agent (Lorusso et al, 1998) or in combination with cisplatin, carboplatin or taxanes (Li et al, 2005; Von Der Maase et al, 2005), has proven effective against metastatic bladder lesions, yielding $27-38 \%$ response rates in different studies. Furthermore, pharmacokinetic studies carried out after intravesical instillation have shown a very low systemic absorption of Gem, indicating this drug as an ideal candidate for intravesical therapy (Cozzi et al, 1999; Laufer et al, 2003).

The aim of this phase II study was to analyse the activity of the Gem treatment scheme that proved to be the most effective in in vitro cell cultures, a system that adequately reproduces the conditions of intravesical clinical treatment. The study was performed on patients refractory to BCG and submitted to transurethral bladder resection (TURB). Secondary end points were time to recurrence, progression and overall tolerability.

\section{MATERIALS AND METHODS}

\section{In vitro studies}

Cell cultures The study was performed on two established bladder cancer cell lines: a commercial cell line (HT1376) with a 
37-h doubling time (obtained from American Type Culture Collection, Rockville, MD, USA) and a cell line (MCR) established in the Biological Laboratory of the Department of Medical Oncology in Forlì (Zoli et al, 2004), with a 48-h doubling time. Cells were maintained as a monolayer in culture medium (DMEM) supplemented with $10 \% \mathrm{FCS}, 100 \mathrm{IU} \mathrm{ml}^{-1}$ penicillin, $100 \mathrm{mg} \mathrm{ml}^{-1}$ streptomycin and $2 \mathrm{~mm}$ L-glutamine and subcultured weekly. All the experiments were performed during exponential cell growth.

Growth inhibition assay Cells were seeded in 96-well, flatbottomed microtitre plates at a density of 10000 cells well $^{-1}$. At $18-24 \mathrm{~h}$ after plating, $100 \mu \mathrm{l}$ of culture medium with or without Gem (kindly supplied by Ely Lilly, Florence, Italy) were added to each well. At the end of drug exposure, cells were fixed with $50 \%$ trichloroacetic acid and stained with $0.4 \%$ sulphorhodamine B (Sigma-Aldrich, St Louis, MO, USA), dissolved in $1 \%$ acetic acid $\left(100 \mu \mathrm{lwell}{ }^{-1}\right)$, and washed with $1 \%$ acetic acid to remove unbound stain. Protein-bound stain was solubilised with $100 \mu \mathrm{l}$ of $10 \mathrm{~mm}$ unbuffered Tris base, and cell density was determined using a fluorescence plate reader (wavelength, 540 or $510 \mathrm{~nm}$ ). The sulphorhodamine B assay was used according to the method of Skehan et al (1990). Cells were exposed to Gem for one or two 1-h periods, the latter spaced out by a 24 - or 48 -h culture in drug-free medium (wash-out). Cells were treated with 1,2 or $3 \mu \mathrm{g} \mathrm{ml}^{-1}$ of Gem, taking into account that the peak plasma level for the drug is $3.2 \mu \mathrm{g} \mathrm{ml}^{-1}$ (Abbruzzese et al, 1991). Control samples were processed in the same way as treated samples but in drug-free medium. Samples were run in octuplet, and each experiment was repeated three times.

Flow cytometry At various observation times, the medium was removed and cells were detached from the flasks by trypsin, washed twice with PBS and stained according to the methods specified below. A FACS Vantage flow cytometer (Becton Dickinson, San Diego, CA, USA), equipped with an argon laser $(488 \mathrm{~nm})$, was used. Data acquisition and analysis were performed using CELLQuest Pro software (Becton Dickinson, San Diego, CA, USA) and ModFit 2.0 (DNA Modelling System, Verity Software House, Inc., Topsham, ME, USA). Samples were run in triplicate and each experiment was repeated three times. Standard errors were below $5 \%$.

Cell cycle perturbations Briefly, samples of $2 \times 10^{5}$ cells were exposed to a $0.1 \mu \mathrm{g} \mathrm{ml}^{-1}$. concentration of Gem for $1 \mathrm{~h}$. Cell cycle distribution was determined immediately after treatment and 24 or $48 \mathrm{~h}$ after drug removal. Control samples were processed in the same way as treated samples but in drug-free medium. At the end of drug exposure, cells were harvested and stained in a solution containing RNase (10 Kunits $\mathrm{ml}^{-1}$; Sigma-Aldrich), NP40 (0.01\%; Sigma-Aldrich) and propidium iodide (PI) $\left(1 \mu \mathrm{g} \mathrm{ml}^{-1}\right.$; SigmaAldrich). After 30-60 min, samples were analysed and expressed as fractions of cells in the different cell cycle phases. Samples were run in triplicate and 10000 events were collected for each replica. Data reported are the average of three experiments, with errors under $5 \%$.

\section{Apoptosis}

Annexin-V assay: After Gem exposure, cells were harvested, washed once in PBS and incubated with $25 \mu \mathrm{ml}^{-1}$ of Annexin V-FITC in binding buffer (Bender MedSystems, Vienna, Austria) for $15 \mathrm{~min}$ at $37^{\circ} \mathrm{C}$ in a humidified atmosphere in the dark. Cells were then washed again in PBS and suspended in binding buffer. Immediately before flow cytometry analysis, PI was added to a final concentration of $5 \mu \mathrm{g} \mathrm{ml}^{-1}$ to distinguish between total apoptotic cells (Ann-V + and PI- or + ) and necrotic cells (Ann-V - and PI + ). For each sample, 15000 events were recorded.
Data analysis: The efficacy of the two Gem exposures according to the different treatment schedules was determined by the $R$ Index (RI) method (Romanelli et al, 1998). Although several methods have been proposed to evaluate the interaction between drugs, as critically analysed by Zoli et al. (2001), most of these are not applicable to drugs with a low cytotoxic effect. We therefore used Kern's method (Kern et al, 1988), modified by Romanelli (Romanelli et al, 1998), to overcome this problem. In brief, the expected cell growth (Sexp), defined as the product of the cell growth observed after exposure to drug $A$ and that observed after drug $B$, and the observed cell growth (Sobs) after the exposure to the combination of $A$ and $B$ were used to construct an index (RI): $R=$ Sexp/Sobs. An $\mathrm{RI}=1$ indicated an additive effect. $\mathrm{RI}$ values higher or lower than 1 indicated synergistic and antagonistic effects, respectively.

\section{In vivo studies}

Inclusion criteria Patients who had disease recurrence (Ta G3, T1 G1-3 TCC) within 6 months of one induction cycle and at least three maintenance cycles of BCG, with no residual disease after TURB, were consecutively enrolled onto this phase II study. Age $\geqslant 18$ years and WHO performance status (PS) $0-1$. Normal upper urinary tract and bladder capacity $>300 \mathrm{ml}$ were documented before recruitment with Uro-CT scan and ultrasonography, respectively. Tumour stage was defined according to the 1997 TNM system (Sobin and Wittekind, 1997) and grading was based on the 1999 WHO classification (Epstein et al, 1998).

Exclusion criteria Previous partial cystectomy, prior pelvic irradiation and clinical evidence of other malignancies or histologically confirmed carcinoma in situ.

Informed consent was obtained before treatment, and patients were required to be accessible for follow-up. The study protocol was approved by the Local Ethics Committee of the participating centres.

Therapeutic and evaluation protocol Two weeks after TURB, the bladder was completely emptied by catheterisation and endovesical instillation was performed with $2000 \mathrm{mg}$ Gem diluted in $50 \mathrm{ml}$ saline solution, without $\mathrm{pH}$ adjustment. The drug was maintained in the bladder for at least $1 \mathrm{~h}$ and then spontaneously eliminated. The instillation was repeated on days 1 and 3 for the six consecutive weeks. No maintenance treatment was planned.

Patients were evaluated for response at the end of treatment and those with negative cystoscopy and cytology underwent close surveillance. Cytological analysis of voided urine and cystoscopy were performed at 3-month intervals for the first year, and every 6 months thereafter. In the event of positive cystoscopy, the lesion was submitted to histological examination. Patients with residual disease discontinued treatment and the subsequent therapeutic approach was defined at the discretion of each patient's physician.

The pre-study clinical evaluation comprised medical history, general physical examination, ECG, Uro-CT scan, chest X-ray and haematological evaluation (including WBC-PLT count, electrolytes and liver and kidney function), and ultrasonography evaluation of bladder capacity. Information on the date of treatment administration, drug doses and chemotherapy-induced side effects was recorded.

Toxicity and complete blood count were evaluated according to NCI Common Toxicity Criteria (version 2.0) on the first day of each cycle of therapy. A clinical, haematological and biochemical assessment for each patient was performed every third week and repeated at the end of treatment.

Gem dose was reduced to $1400 \mathrm{mg}$ in the presence of grade 3 urgency or dysuria in association with grade 3-4 haematuria. The dose reduction was maintained throughout the treatment and instillation was stopped if toxicity persisted. Intravesical treatment 
was discontinued if the patient developed febrile neutropaenia

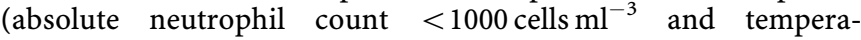
ture $\geqslant 38^{\circ} \mathrm{C}$ ), documented bacteraemia in the presence of neutropaenia, grade 3 or 4 neutropaenia/thrombocytopaenia, bilirubin $>1.5 \times$ upper limit of normal or transaminase $>3 \times$ upper limit of normal. Therapy was delayed by one week in the event of grade 3 bladder toxicity.

\section{Statistical analysis}

For the primary objective of the clinical study, that is, the evaluation of Gem activity, sample size was determined using the optimum Simon's two-stage design (Simon, 1989). 'Response' was defined as the lack of residual disease at 6 months, certified by cytological and endoscopic examinations. Positive urinary cytology, new lesions (superficial or infiltrating), nodal involvement and systemic diffusion were considered a 'no-response'. The study was sized to refuse 'response' rates of $40 \%\left(P_{0}\right)$ and to provide a significance level of 0.10 with a statistical power of $90 \%$ in assessing the activity of the regimen as a $60 \%$ 'response' rate $\left(P_{1}\right)$. The upper limit for first-stage drug rejection was 11 'responses' in the 28 assessable patients. The upper limit of second-stage rejection was 20 'responses' within a total of 41 assessable patients.

Event-free survival (EFS) was defined as the interval between the date of the first endovesical instillation and the first unfavourable event, superficial disease, progression to infiltrating disease or the last visit. Event-free survival was estimated by the Kaplan-Meier method. Ninety-five percent confidence intervals (95\% CI) for 'response' and EFS estimates were calculated.

\section{RESULTS}

\section{In vitro studies}

A cytostatic dose-dependent effect was produced by Gem after all treatment schemes. The effect induced by a 1-h exposure to Gem significantly increased $(P<0.05)$ after a 24 - and 48 -h culture in drug-free medium (wash-out) at all tested concentrations. A generally higher sensitivity of MCR than HT1376 cells was observed (Figure 1). Moreover, the analysis of the types of interaction between the two 1-h exposures to Gem showed an antagonistic effect on cell growth when these were consecutive. Conversely, an additive interaction in HT1376 and synergistic activity in MCR were observed when the two exposures were spaced out by a $24-\mathrm{h}(\mathrm{RI}=1.5)$ or $48-\mathrm{h}(\mathrm{RI}=1.6)$ wash-out.

Cell cycle perturbations and induction of apoptosis were analysed in an attempt to find an explanation for the different types of interaction. After a 1-h exposure to Gem, we observed a statistically significant increase of cells in G0-G1 phases (67\% in treated $v s 46 \%$ in untreated samples, $P<0.05)$, together with a similar decrease in both $\mathrm{S}(25 \%$ vs $40 \%, P<0.05)$ and $\mathrm{G} 2-\mathrm{M}(7.3 \%$ vs $14 \%, P<0.05)$ phases. These perturbations were still present $24 \mathrm{~h}$ after Gem removal and the block in G0-G1 phases recovered only after $48 \mathrm{~h}$, after which $S$ phase, the antimetabolite's target, was repopulated (Table 1 ).

Gem treatment induced apoptosis in about one-third of tumour cells. More specifically, $25 \%$ of apoptotic cells were observed after a 1-h exposure to Gem followed by a 72-h culture in drug-free medium, increasing to $35 \%$ following the sequential treatment (1-h $\mathrm{Gem} \rightarrow 48$-h wash-out $\rightarrow 1$-h $\mathrm{Gem} \rightarrow 24$-h wash-out). Conversely, the percentage of necrotic cells never exceeded $5 \%$.

\section{In vivo studies}

Forty-one consecutive patients were enrolled onto the study by the Urology Department of Forlì and the Oncology Department of Cattolica. Forty patients completed treatment and were evaluable for clinical end points and one patient was lost to follow-up immediately after registration. All but one of the patients were males, and age ranged from 40 to 87 years (median 66 years). About two-thirds presented a monofocal lesion, 90\% had T1 tumours and more than $95 \%$ had G2-3 lesions (Table 2).

\section{Activity}

In accordance with a Simon's two-step design, we continued recruitment into the second step, having observed more than 11
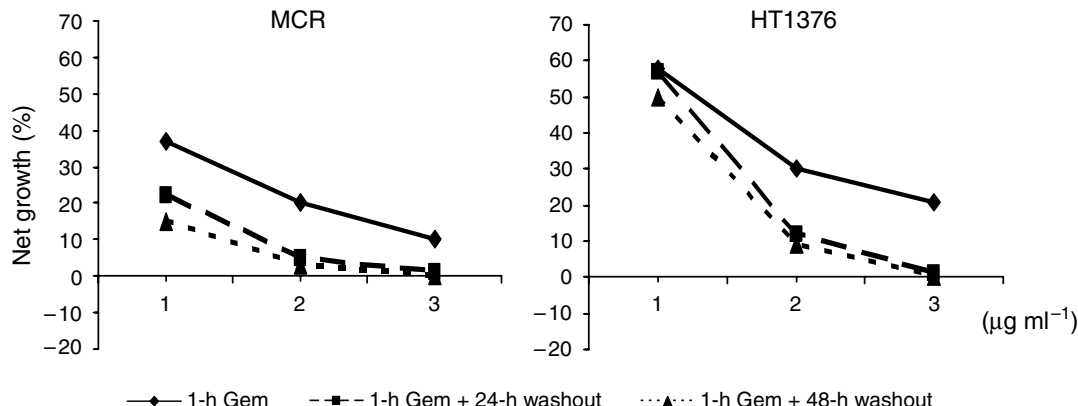

Figure I Dose-response cytotoxicity of Gem in bladder cancer cell lines after different treatment schemes.

Table I Distribution of cells in the different cycle phases after exposure to Gem $\left(\mid \mu \mathrm{g} \mathrm{ml}^{-1}\right)$

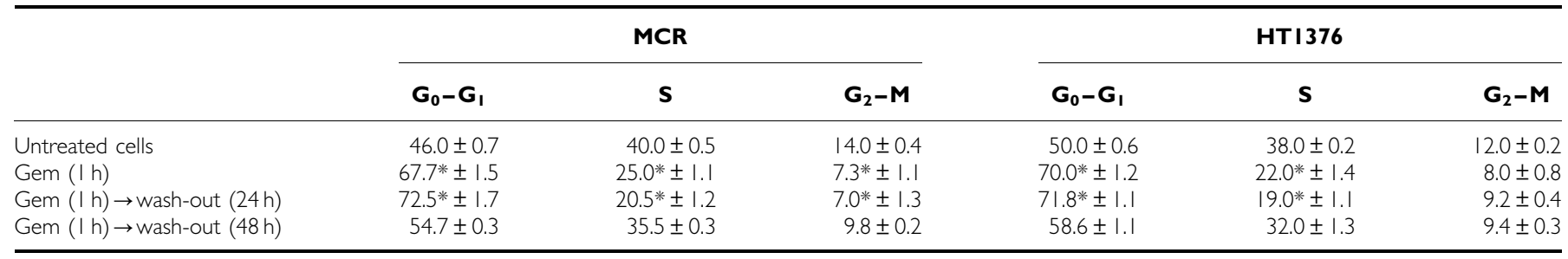

Data represent mean percentage values \pm s.d. $* P<0.05$ 
responses in the first 28 enrolled patients. On the basis of the primary end point of the study, 38 of the $40(95 \%)$ evaluable patients (95\% CI: $83.1-99.4 \%)$ obtained a 'response' with respect to the minimum expected rate of $60 \%$.

Specifically, a 'response' was observed in 21 of the 23 high-risk tumour patients (91\%; 95\% CI: $72.0-98.9 \%$ ) and in all 17 intermediate-risk patients. The probability of event-free survival for the entire case series was around $80 \%$ at 1 year and $66 \%$ at 2.5 years (Figure 2). At a median follow-up of 28 months, superficial recurrences had been observed in 14 patients. Among these, four relapsed with downstaged $(\mathrm{T})$ disease, three exhibited a lower grade $(G)$ lesion and three had a reduction in both $T$ and $G$. Finally, two monofocal lesions presented as multifocal at relapse, while the inverse situation was observed in one patient. Only two

Table 2 Patient and tumour characteristics

\begin{tabular}{|c|c|c|}
\hline & $n$ & $\%$ \\
\hline \multicolumn{3}{|l|}{ Gender } \\
\hline Male & 38 & 92.5 \\
\hline Female & 2 & 5.0 \\
\hline \multicolumn{3}{|l|}{ Age (years) } \\
\hline$<60$ & 10 & 25.0 \\
\hline $60-74$ & 17 & 42.5 \\
\hline$\geqslant 75$ & 13 & 32.5 \\
\hline \multicolumn{3}{|l|}{ Focality } \\
\hline Monofocal & 25 & 62.5 \\
\hline Mulltifocal & 15 & 37.5 \\
\hline \multicolumn{3}{|l|}{ Stage } \\
\hline $\mathrm{Ta}$ & 4 & 10.0 \\
\hline TI & 36 & 90.0 \\
\hline \multicolumn{3}{|l|}{ Grading } \\
\hline GI & 2 & 5.0 \\
\hline G2 & 21 & 52.5 \\
\hline G3 & 17 & 42.5 \\
\hline \multicolumn{3}{|l|}{ PS (WHO) } \\
\hline 0 & 36 & 90.0 \\
\hline 1 & 4 & 10.0 \\
\hline
\end{tabular}

patients relapsed at 5 and 6 months and both subsequently underwent cystectomy (Table 3 ).

\section{Toxicity}

Systemic toxicity was very low, with no evidence of alterations in biochemical profiles. Hyperthermia $\geqslant 38^{\circ} \mathrm{C}$ was observed in only one patient and required a Gem dose reduction. Hyperthermia $<38^{\circ} \mathrm{C}$ was observed in six cases and was well controlled by antipyretics. According to NCI-CTC criteria, 10 cases of grade 1 and 27 cases of grade 2 dysuria (pollakiuria and/or stranguria with or without urgency) were observed. Good control of symptoms was obtained in these patients with the administration of anticholinergic drugs. Grade 3 dysuria caused a delay in therapy in only three patients and was resolved with anticholinergics. A consequent reduction in Gem dose was required. Haematuria was never observed.

Table 3 Variations in Gem-induced pathological characteristics in relapsed patients

\begin{tabular}{llll}
\hline Focality & T & G \\
\hline
\end{tabular}

Time to

Patient recurrence Treatment at

code (months) Pre Post Pre Post Pre Post relapse

\begin{tabular}{|c|c|c|c|c|c|c|c|c|}
\hline 03 & 12 & $M$ & $M$ & I & । & 3 & 2 & Cystectomy (+CIS) \\
\hline 06 & 13 & $\mathrm{~m}$ & $M$ & । & । & 2 & 2 & TURB \\
\hline 07 & 31 & $M$ & $\mathrm{~m}$ & । & । & 2 & 2 & TURB \\
\hline 09 & 31 & $\mathrm{~m}$ & $\mathrm{~m}$ & l & a & 3 & 2 & TURB \\
\hline 16 & 10 & $M$ & $M$ & l & । & 2 & 2 & Cystectomy \\
\hline 19 & 6 & $\mathrm{~m}$ & $M$ & i & a & 2 & 2 & TURB \\
\hline 26 & 19 & $M$ & $M$ & a & a & 2 & I & TURB \\
\hline 29 & 19 & $\mathrm{~m}$ & $\mathrm{~m}$ & a & $\mathrm{a}$ & 2 & 2 & TURB \\
\hline 30 & 14 & $\mathrm{~m}$ & $\mathrm{~m}$ & । & $\mathrm{a}$ & 3 & । & TURB \\
\hline 32 & 8 & $\mathrm{~m}$ & $\mathrm{~m}$ & a & $\mathrm{a}$ & 2 & 2 & TURB \\
\hline 34 & 13 & $M$ & $M$ & I & । & 2 & 2 & TURB \\
\hline 37 & 8 & $M$ & $M$ & I & a & 2 & I & TURB \\
\hline 38 & 5 & $M$ & $M$ & $\mathrm{a}$ & $\mathrm{a}$ & 3 & 2 & TURB \\
\hline 39 & II & $\mathrm{m}$ & $\mathrm{m}$ & I & I & 2 & 2 & TURB \\
\hline
\end{tabular}

$T=$ tumour stage; $G=$ tumour grade; $M=$ multifocal lesion; $m=$ monofocal lesion; $\mathrm{CIS}=$ carcinoma in situ; $\mathrm{TURB}=$ transurethral bladder resection.

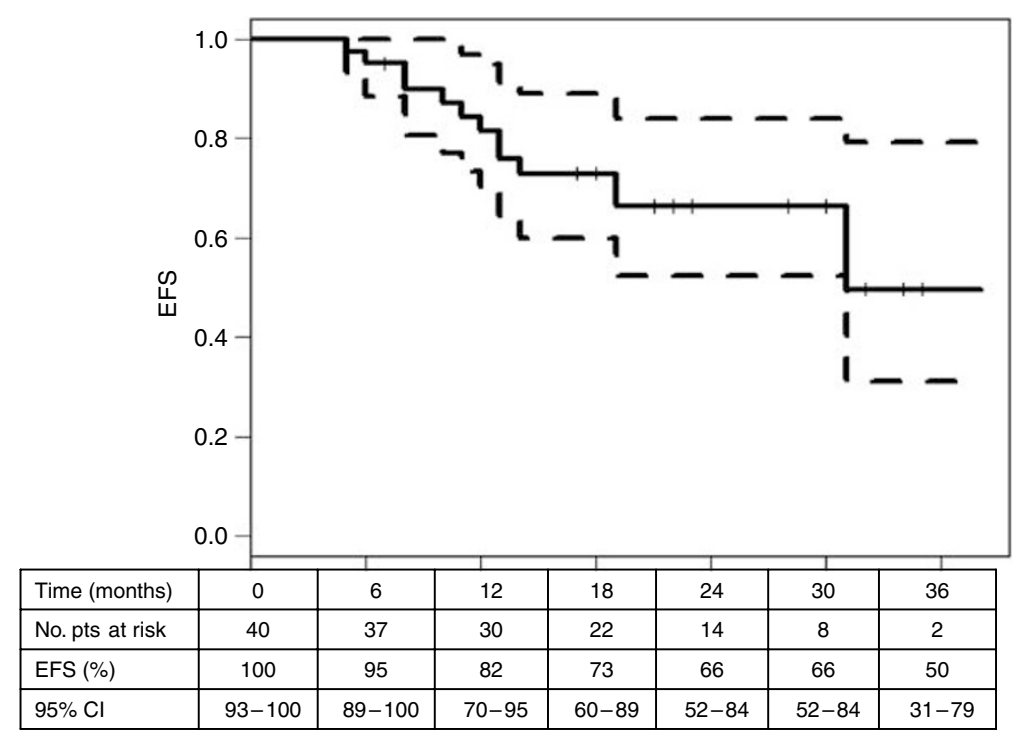

Figure 2 Event-free survival and $95 \% \mathrm{Cl}$ curves. 


\section{DISCUSSION}

Although the standard adjuvant treatment for superficial bladder cancer at high-risk of recurrence is intravesical BCG, the role of this therapy is somewhat controversial for intermediate-risk tumours due to the high frequency of side effects and the uncertain risk/benefit ratio. Moreover, there is still no recommended standard therapy for patients relapsing after conventional intravesical treatments. New treatment options are therefore needed, and Gem represents a promising drug.

In a phase I study by Dalbagni et al (2002), patients with BCGrefractory bladder carcinoma were treated with biweekly instillations of Gem at scalar doses up to $20 \mathrm{mg} \mathrm{ml}^{-1}$ for 6 weeks. This schedule produced a $50 \%$ complete remission rate, with acceptable local and systemic toxicity. A lower toxicity was reported by Laufer et al (2003) in a dose-finding and pharmacokinetic study using weekly Gem instillations at scalar doses up to $40 \mathrm{mg} \mathrm{ml}^{-1}$ for 6 weeks. At the maximum dose, the plasmatic level of Gem was low and grade 2 dysuria was observed in only one patient.

In our clinical protocol, in which the Gem intravesical treatment scheme was derived from preclinical studies designed to evaluate the cytotoxic activity of different Gem schedules, all but one patient concluded the planned treatment. Moreover, moderate urinary toxicity and no haematological side effects were observed, notwithstanding the biweekly instillations, each of $20 \mathrm{mg} \mathrm{ml}^{-1}$ of Gem, for 6 weeks.

With regard to Gem activity, some phase II studies (De Berardinis et al, 2004; Gontero et al, 2004; Campodonico et al, 2005) assessed its ablative effect on a single marker lesion left in the bladder after complete resection of all other lesions. In these studies, Gem, administered weekly for 6 weeks, produced a response rate of about $50 \%$ with a very low frequency of systemic and local toxicity, which generally did not exceed grade I.

The activity of Gem has also been investigated in BCG-refractory patients following either weekly or biweekly administration. In Bartoletti et al's (2005) study, Gem given once a week for 6 weeks was associated with a $74 \%$ and $13 \%$ recurrence-free survival in

\section{REFERENCES}

Abbruzzese JL, Grunewald R, Weeks EA, Gravel D, Adams T, Nowak B, Mineishi S, Tarassoff P, Satterlee W, Raber MN (1991) A Phase I clinical, plasma, and cellular pharmacology study of gemcitabine. J Clin Oncol 9: $491-498$

Bartoletti R, Cai T, Gacci M, Giubilei G, Viggiani F, Santelli G, Repetti F, Nerozzi S, Ghezzi P, Sisani M, TUR (Toscana Urologia) Group (2005) Intravesical gemcitabine therapy for superficial transitional cell carcinoma: results of a phase 2 prospective multicenter study. Urology 66: $726-731$

Campodonico F, Canepa G, Capponi G, Bozzo L, Maffezzini M (2005) Intravesical gemcitabine in recurrent superficial bladder carcinoma: preliminary results on ablative efficacy and tolerability. Anticancer Res 25: $2381-2384$

Cozzi PJ, Bajorin DF, Tong W, Nguyen H, Scott J, Heston WD, Dalbagni G (1999) Toxicology and pharmacokinetics of intravesical gemcitabine: a preclinical study in dogs. Clin Cancer Res 5: 2629-2637

Dalbagni G, Russo P, Bochner B, Ben-Porat L, Sheinfeld J, Sogani P, Donat MS, Herr HW, Bajorin D (2006) Phase II trial of intravesical gemcitabine in bacille Calmette-Guerin-refractory transitional cell carcinoma of the bladder. J Clin Oncol 24: 2729-2734

Dalbagni G, Russo P, Sheinfeld J, Mazumdar M, Tong W, Rabbani F, Donat MS, Herr HW, Sogani P, dePalma D, Bajorin D (2002) Phase I trial of intravesical gemcitabine in Bacillus Calmette-Guerin-refractory transitional-cell carcinoma of the bladder. J Clin Oncol 20: 3193-3198

De Berardinis E, Antonini G, Peters GJ, Loves WJ, Van der Born K, Codacci-Pisanelli G, Di Silverio F (2004) Intravesical administration of gemcitabine in superficial bladder cancer: a phase I study with pharmacodynamic evaluation. BJU Int 93: 491 - 494

Epstein J, Amin M, Reuter V, Mostofi F (1998) The World Health Organization/International Society of Urological Pathology consensus patients with intermediate- and high-risk lesions, respectively, at a median follow up of 13.6 months. The usefulness of the antimetabolite was also confirmed by Kohjimoto in a small series of BCG-refractory patients following biweekly administration (Kohjimoto et al, 2005).

In a recent phase II study, 30 BCG-refractory patients were treated biweekly with $2000 \mathrm{mg}$ Gem diluted in $100 \mathrm{ml}$ saline solution for 3 weeks, with each course separated by 1 week's rest (Dalbagni et al, 2006). The complete disappearance of all evidence of disease was obtained in $50 \%$ of patients and $21 \%$ were recurrence-free at 1 year.

In the present study, following a biweekly Gem administration for 6 weeks, 80 and $66 \%$ of patients were event-free at the 1 - and 2.5-year follow up, respectively, with fairly similar rates in intermediate- and high-risk tumour subgroups. It is also noteworthy that at relapse, lesions were frequently downstaged and only two patients required cystectomy.

These important therapeutic results, which could at least delay the need for invasive cystectomy, were associated with very modest toxicity, underlying the importance of the experimentally derived scheme.

In conclusion, further clinical studies are now needed to verify the efficacy of this Gem schedule in an adjuvant setting, and more preclinical research is warranted to explore and identify the most effective Gem-containing combinations to evaluate in randomised phase II-III studies.

\section{ACKNOWLEDGEMENTS}

We thank Herbert Hanitszch, Paola Lilli and Carlo Vivacqua of the Department of Urology, Morgagni-Pierantoni Hospital in Forlì for their precious assistance, and Gráinne Tierney for editing the manuscript. This work was supported by Istituto Oncologico Romagnolo, Forlì, and by Consiglio Nazionale della Ricerca/Ministero della Istruzione, Università e Ricerca (CNR/MIUR - Progetto Strategico), grant no. 02.00278.ST87, Rome, Italy. classification of urothelial (transitional cell) neoplasms of the urinary bladder. Bladder Consensus Conference Committee. Am J Surg Path 22: $1435-1448$

Gontero P, Casetta G, Maso G, Sogni F, Pretti G, Zitella A, Frea B, Tizzani A (2004) Phase II study to investigate the ablative efficacy of intravesical administration of gemcitabine in intermediate-risk superficial bladder cancer (SBC). Eur Urol 46: 339-343

Kern DH, Morgan CR, Hildebrand-Zanki SU (1988) In vitro pharmacodynamics of 1-beta-D-arabinofuranosylcytosine: synergy of antitumor activity with cis-diamminedichloroplatinum(II). Cancer Res 48: 117-121

Kohjimoto Y, Iba A, Shintani Y, Uekado Y, Shinka T (2005) Treatment of Bacillus Calmette-Guerin refractory superficial bladder cancer: further intravesical therapy. Hinyokika Kiyo 51: 533-538

Laufer M, Ramalingam S, Schoenberg MP, Haisfield-Wolf ME, Zuhowski EG (2003) Intravesical gemcitabine therapy for superficial transitional cell carcinoma of the bladder: a phase I and pharmacokinetic study. J Clin Oncol 21: 697-703

Li J, Juliar B, Yiannoutsos C, Ansari R, Fox E, Fisch MJ, Einhorn LH, Sweeney CJ (2005) Weekly paclitaxel and gemcitabine in advanced transitional-cell carcinoma of the urothelium: a phase II Hoosier Oncology Group study. J Clin Oncol 20: 1185-1191

Lorusso V, Pollera CF, Antimi M, Luporini G, Gridelli C, Frassineti GL, Oliva C, Pacini M, De Lena M (1998) A phase II study of gemcitabine in patients with transitional cell carcinoma of the urinary tract previously treated with platinum. Italian Co-operative Group on Bladder Cancer. Eur J Cancer 34: 1208 - 1212

Malmstrom P (2000) Improved patient outcomes with BCG immunotherapy vs. chemotherapy: Swedish and worldwide experience. Eur Urol 37: 16-20 Malmstrom PU, Wijkstrom H, Lundhholm C, Wester K, Busch C, Norlen BJ (1999) Five-year followup of a randomized prospective study comparing 
mitomycin $\mathrm{C}$ and Bacillus Calmette-Guerin in patients with superficial bladder carcinoma. Swedish-Norwegian Bladder Cancer Study Group. J Urol 161: 1124-1127

Oosterlinck W, Van der Meijden A, Sylvester R, Böhle E, Rintala E, Solsona Narvón E, Lovel B (2006) European Association of Urology (EAU): Guidelines on TaT1 (non muscle-invasive) bladder cancer (2006) - www. uroweb.org/fileadmin/user_upload/Guidelines/05\%20TaT1\%20Bladder\%20 Cancer.pdf

Romanelli S, Perego P, Pratesi G, Carenini N, Tortoreto M, Zunino F (1998) In vitro and in vivo interaction between cisplatin and topotecan in ovarian carcinoma systems. Cancer Chemother Pharmacol 41: $385-390$

Simon R (1989) Optimal two-stage designs for phase II clinical trials. Control Clin Trials 10: 1-10

Skehan P, Storeng R, Scudiero D, Monks A, McMahon J, Vistica D, Warren JT, Bokesch H, Kenney S, Boyd MR (1990) New colorimetric cytotoxic assay for anticancer drug screening. J Natl Cancer Inst 13: $1107-1112$
Sobin LH, Wittekind CH (eds) (1997) TNM Classification Malignant Tumours 5th edn. John Wiley \& Sons, Inc: New York

Sylvester RJ, van der Meijden AP, Lamm DL (2002) Intravesical bacillus Calmette-Guerin reduces the risk of progression in patients with superficial bladder cancer: a meta-analysis of the published results of randomized clinical trials. J Urol 168: $1964-1970$

Von Der Maase H, Seugelov L, Roberts JT, Roberts JT, Ricci S, Dogliotti L, Oliver T, Moore MJ, Zimmermann A, Arning M (2005) Long-term survival of a randomized trial comparing gemcitabine plus cisplatin with methotrexate-vinblastine-doxorubicin plus cisplatin in patients with bladder cancer. J Clin Oncol 23: 4602-4608

Zoli W, Ricotti L, Tesei A, Barzanti F, Amadori D (2001) In vitro preclinical models for a rational design of chemotherapy combinations in human tumors. Crit Rev Oncol Hematol 37: 69-82

Zoli W, Ricotti L, Tesei A, Ulivi P, Gasperi Campani A, Fabbri F, Gunelli R, Frassineti GL, Amadori D (2004) Schedule-dependent cytotoxic interaction between epidoxorubicin and gemcitabine in human bladder cancer cells in vitro. Clin Cancer Res 10: $1500-1507$ 